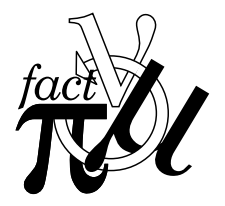

NuFact Note 100

October 31, 2001

\title{
Multiple pion beam collection
}

\author{
B. Autin, A. Verdier \\ Cern, CH-1211 Geneve 23, Switzerland
}

\begin{abstract}
In neutrino factories, the proton beam power is of the order of 4 MW. The target and collecting devices work in rather extreme conditions if the whole proton beam is dumped on a single target. The situation is improved if the proton beam is directed on several targets, the deposited power is then divided by the number of targets. A scheme for collecting four pion beams is proposed using alternating gradient quadrupoles.
\end{abstract}

funneling. 


\section{Introduction}

The proton drivers of neutrino factories deliver beams whose power exceeding $1 \mathrm{MW}$ and it is generally admitted that raising the proton power to $4 \mathrm{MW}$ would provide the muon beam required for the most precise experiments. In the case of the CERN scheme, the protons are distributed over 140 bunches forming a train of $3 \mu \mathrm{s}$ repeated at a frequency of $50 \mathrm{~Hz}$. Compared with the few short bunches delivered by a synchrotron, a long train is favorable for the target because the themperature increase is reduced. On the other hand, the horn which collects the pions using a current of $300 \mathrm{kA}$ has to pulse at a high repetition rate and its feasibility is still unproven. Replacing a single target-collector system by $n$ identical systems would divide both power and repetition rate per system by $n$. The technique of recombination of several beams is called "funneling". It has been used for the transfer of the four beams of the PS booster to the CPS accelerator at CERN [1], it is proposed for the drive beam of CLIC [2] and it was suggested for increasing the muon intensity in the decay ring of a neutrino factory or of a muon collider [3]. The idea is resumed here for four pion beams. The scheme is described in Section 1, the optics in Section 2 and the magnet parameters in Section 3.

\section{Recombination of four pion beams}

The recombination of four pion beams is illustrated in Fig. 1. The protons coming from the driver are sequencially switched by a deflection magnet to four targets that can be labeled left, up, right and down. Left and right targets on one hand, up and down targets on the other hand lie in the horizontal and vertical planes respectively. The pions emerging from each target are collected by a horn. Both target and horn point towards the vertex of a cone. The pions are then deflected in a string of dipoles and quadrupoles and finally travel on the cone axis with the same spacing as the proton bunches. At the end of the channel, the orbit dispersion introduced by the deflecting dipoles is made zero in order to cancel the largest chromatic aberration. The free space left inside the collection cone could be used to dump useless particles and especially the primary protons. The magnets are powered with alternating polarities so that the focusing is the same for all the beams and the deflections act specifically on each beam (Fig. 3). The current is shown AC but would likely be pulsed to save power dissipation in 


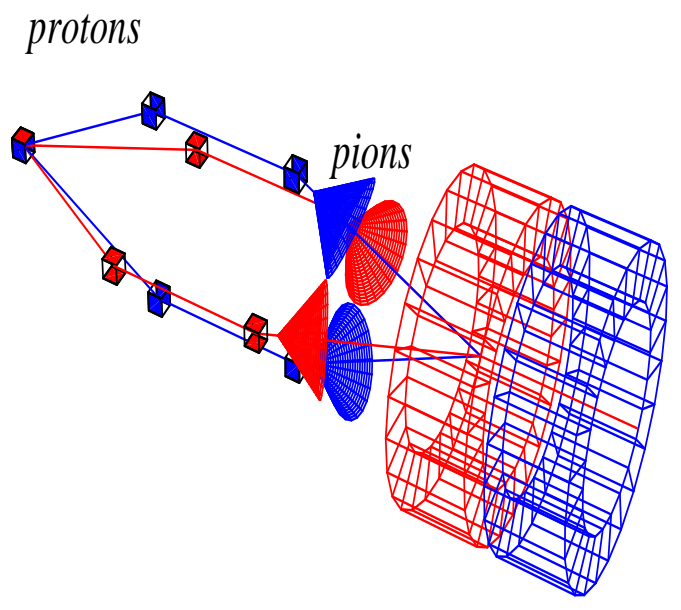

Figure 1: Scheme of the four horns arrangement.

the magnets.

\section{Optics}

The basic module is an anti-symmetrical quadruplet of quadrupoles which leave a round beam invariant. The $\beta$-functions are the sames in both planes at the entrance and the exit and their derivatives are zero. The antisymmetry guarantees that the maximum $\beta$ 's are equal in both planes. Moreover, the channel can be matched to a downstream solenoidal channel [4] which is necessary to reduce the muon losses during the pion decay.

In its simplest form, the quadruplet is made of two doublets which map a waist to a cross-over [5]. In a more detailed study, it turns out that this system contains five free parameters: three lengths and two gradients. It can be checked numerically that generally three parameters are necessary to match the $\beta$ 's to specified value and zero $\alpha$ 's. For special cases, when the ratio between distances between quadrupoles is below about 3 , the two gradients are sufficient and there are two solutions for the gradients.

The horn radius is $0.2 \mathrm{~m}$ and we aim at collecting a beam with a $0.01 \mathrm{rad} . \mathrm{m}$ 


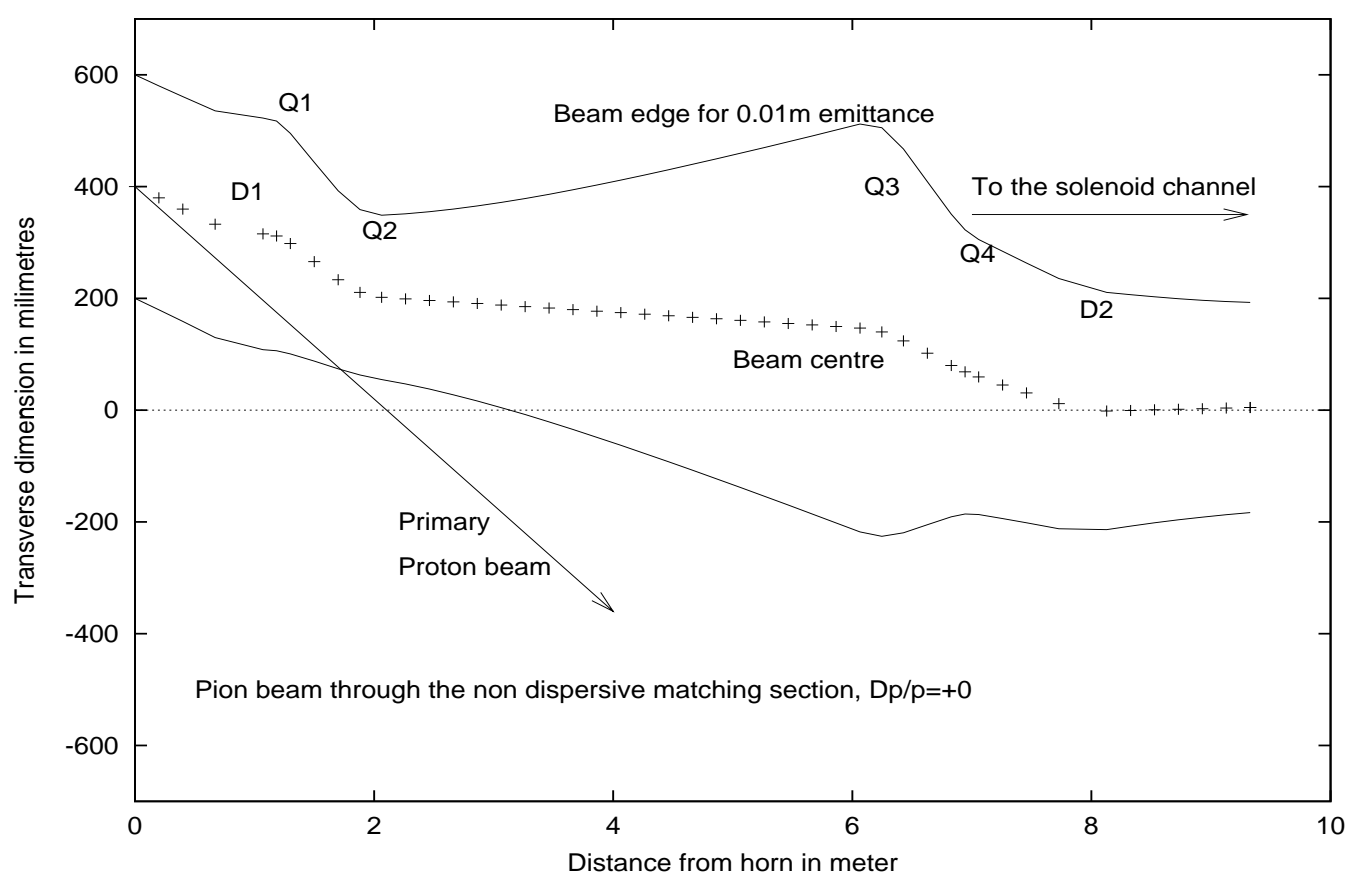

Figure 2: Pion beam in the horizontal plane in the matching section between the horn (on the left) and the RF matching section (on the right). The horn centre is $400 \mathrm{~mm}$ from the quadrupole axis.

maximum emittance for a central kinetic energy of 200MeV (normalised maximum emittance of $0.0271 \mathrm{rad} . \mathrm{m})$. We assume that the beam emitted by the horn has a waist at its exit. Consequently the value of the $\beta$-function at the exit of the horn must be $4 \mathrm{~m}$ and its derivative zero.

An example of optics functions in the matching section is shown on figure 4.

The deflection of the beam is done by both quadrupoles and dipoles. The beam centre has to arrive at the end of the section with a zero slope on the axis, as shown in figure 4 . The dispersion function and its derivative must also be zero, so that the off-momentum particle arrive under about the same condition as the reference particles. This necessitates four parameters.

The triplet optics contain two free parameters, therefore two other parameters are necessary to match the system. As we are dealing with trajectories and dispersion, dipole fields are obvious candidates. Several places have been considered for the dipoles. It has been determined numerically that the most 


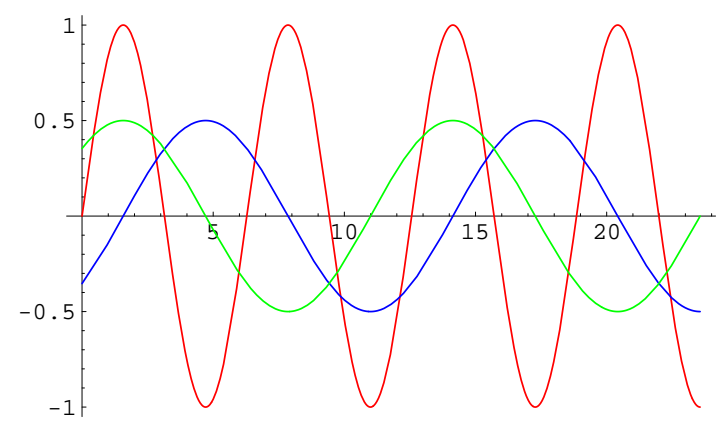

Figure 3: Variation of current with time in quadrupoles (red), horizontal dipoles (blue) and vertical dipoles (green).

efficient dipoles are located in front of the first quadrupole (D1 in front of Q1 in figure 4) and at the end of the section. An additional parameter is the slope of the beam at the entrance, its transverse position being fixed to $0.4 \mathrm{~m}$ (four horns compactly assembled together).

The optics computations have been made with the MAD program [6]. However there is a problem with the definition of the dispersion around a non-zero orbit. This is why a procedure using a numerically computed value of the dispersion has been used.

The matching section shown above is not unique. There are yet two free parameters, e.g. distances between quadrupoles, to be adjusted for a final optimisation.

The effect of dispersion cancellation can be seen on figure 5. The deviation of the beam centre from the axis is negative for both positive and negative momentum deviation. The beam goes out almost parallel to the axis, with a deviation much smaller than the beam size for both relative momentum deviations -0.25 and 0.50 .

The beam size at the exit varies considerably with the momentum deviation. The optics functions associated with different momentum deviations are shown in table 1.

All the above considerations are valid for the case of par-axial conditions and no space-charge, which is not our case. This needs further investigations. 


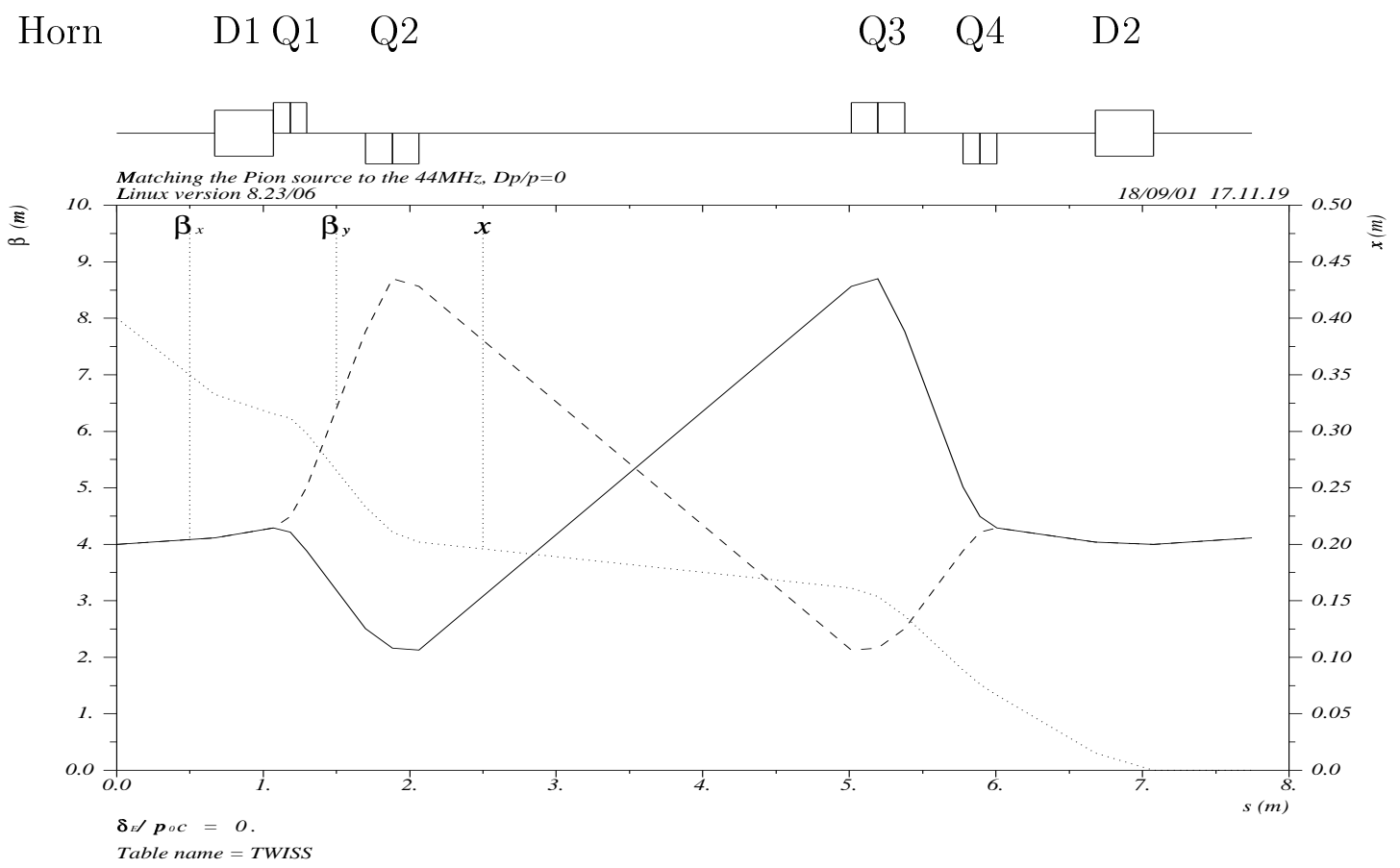

Figure 4: Optics functions and central trajectory in the matching section between the horn (on the left) and the RF matching section (on the right). The elements labelled D are dipoles, the elements labelled Q are quadrupoles.

\section{Hardware issues}

The quadrupole apertures have to be of the order of $0.6 \mathrm{~m}$ to accommodate the off-momentum particles. A design similar to that of super-conducting quadrupoles could be used. The field in the dipoles is smaller than $0.3 \mathrm{~T}$ for a length of $0.4 \mathrm{~m}$. The magnet parameters are given in table 2 . The primary proton beam passes through the first two quadrupoles. Its incidence angle on the targets has to be adjusted so that a beam dump can be installed in the centre of the matching section (see figure 2). 

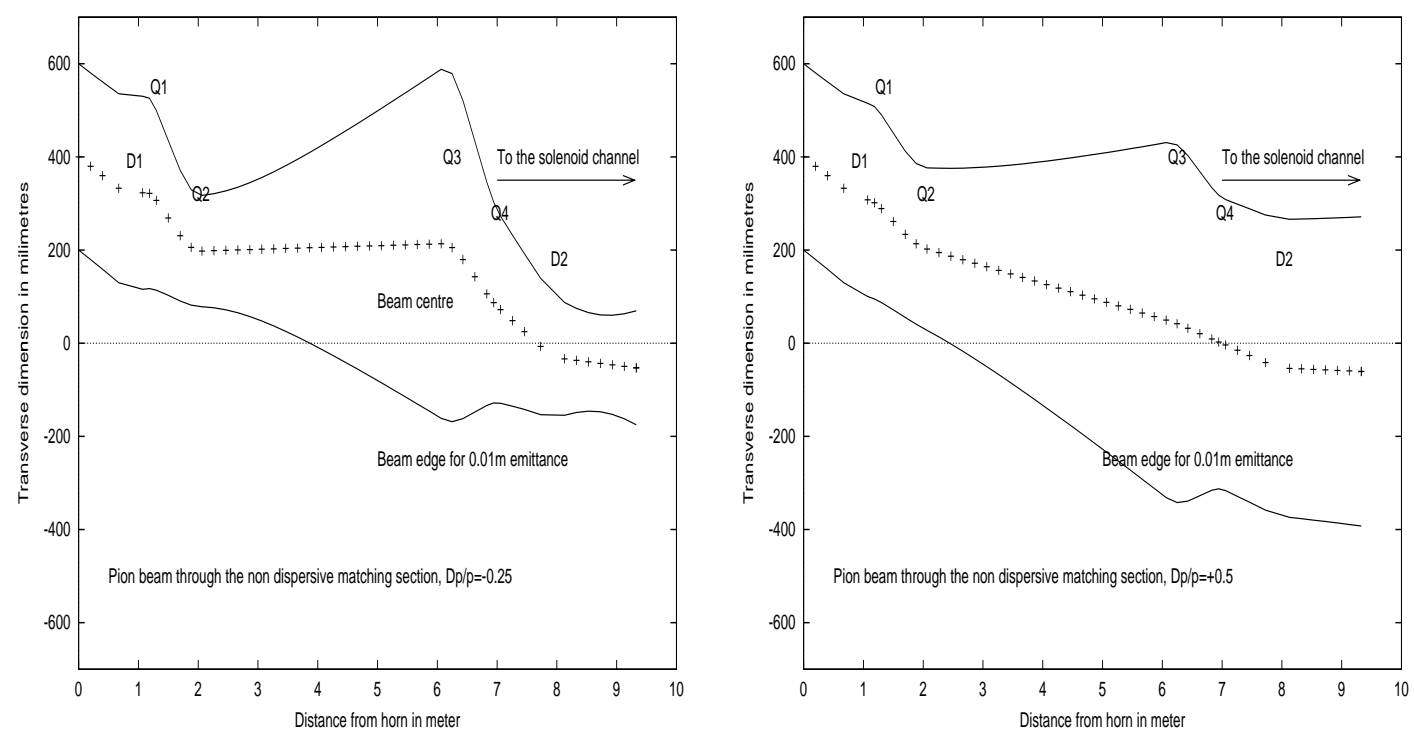

Figure 5: Off-momentum pion beam in the matching section, in the horizontal plane, between the horn (on the left) and the RF matching section (on the right). The relative momentum deviation is 0.25 for the upper graph and 0.5 for the lower graph.

\begin{tabular}{|c|c|c|c|}
\hline Momentum deviation & -0.25 & 0 & 0.5 \\
\hline$\beta_{x, \text { exit }} / \mathrm{m}$ & 1.88 & & 8.18 \\
$\beta_{y, \text { exit }} / \mathrm{m}$ & 7.07 & & 9.03 \\
$\mathrm{x}($ exit $) / \mathrm{mm}$ & -32.4 & 0 & -35.6 \\
path length dif./mm & +14.2 & 0 & -3.76 \\
\hline
\end{tabular}

Table 1: Off-momentum optics functions for the matching section.

\begin{tabular}{|c|c|c|c|}
\hline Magnet & length/m & field/T & gradient/Tm \\
\hline Dipole D1 & 0.4 & 0.271 & $/$ \\
Quadrupole Q1,Q4 & 0.92 & $/$ & 1 \\
Quadrupole Q2,Q3 & 1.37 & $/$ & 1 \\
Dipole D2 & 0.4 & 0.183 & $/$ \\
\hline
\end{tabular}

Table 2: Magnet parameters for the matching section. 


\section{References}

[1] J.P. Delahaye, La recombinaison des faisceaux issus des quatre anneaux du CERN PS Booster, CERN/PS/BR 79-12 (1979).

[2] J.P. Delahaye et al., CLIC, a 0.5 to $5 \mathrm{TeV} e^{+} e^{-}$collider, CERN/PS/98009(LP).

[3] B. Autin, A tentative layout of muon machines on the CERN site, in Prospective Study of Muon Storage Rings at CERN (B. Autin, A. Blondel and J. Ellis editors), CERN 99-02, ECFA 99-197 (1999).

[4] B. Autin, K. Bongardt, J. Pasternak, Bunch compression for longitudinal muon collection, These Proceedings.

[5] B. Autin, C. Carli, T. d'Amico, O. Grobner, M. Martini, E. Wildner, BeamOptics, A program for analytical beam optics, CERN 98-06 (1998).

[6] H. Grote and F.C. Iselin, The MAD program (Methodical Accelerator Design) version 8.16, User's reference manual, CERN/SL/90-13(AP), (rev. 4) (March 27, 1995). 